\title{
Concentration of surfactant protein D, Clara cell protein CC-16 and IL-10 in bronchoalveolar lavage (BAL) in patients with sarcoidosis, hypersensivity pneumonitis and idiopathic pulmonary fibrosis
}

\author{
Wojciech Kucejko, Elżbieta Chyczewska, Wojciech Naumnik, Maria Ossolińska \\ Department of Lung Diseases and Tuberculosis, Medical University of Białystok, Białystok, Poland
}

\begin{abstract}
The process of interstitial inflammation, often chronic, goes fluently from alveolitis through granuloma formation to irreversible fibrosis and lung remodeling. Eventually, the loss of functional alveolar units leads to chronic respiratory failure. The pneumoproteins (e.g. SP-D, CC-16) are considered to be markers of interstitial inflammation. We measured BAL concentration of SP-D, CC-16 and IL-10 in patients with sarcoidosis (27), IPF (7) and HP (9). The level of each marker was determined by ELISA specific kit. We found the highest SP-D and CC-16 BAL concentration in patients with the III stage of sarcoidosis $(96,67 \mathrm{ng} / \mathrm{ml}$ and 31,78 ng/ml, respectively). The lowest SP-D concentration was observed in patients with IPF $(76,49 \mathrm{ng} / \mathrm{ml})$, and the lowest CC-16 concentration in patients with HP $(21,39 \mathrm{ng} / \mathrm{ml})$. The differences were not statistically significant. In the group of the III stage of sarcoidosis higher SP-D levels were related to higher BAL cytosis and higher percentage of BAL neutrophils, just the opposite as in the IPF and HP group. In the III stage of sarcoidosis and HP, the lower SP-D levels, the lower FEV1 and VC values. The results show, that in acute interstitial inflammation with larger parenchyma engagement (III stage of sarcoidosis) the levels of SP-D were higher then in chronic interstitial inflammation (IPF).
\end{abstract}

Key words: surfactant protein D, Clara cell protein, IL-10, sarcoidosis, pulmonary fibrosis, interstitial lung disease, allergic extrinsic alveolitis.

\section{Introduction}

Mostly chronic course of the interstitial lung diseases leads to pulmonary fibrosis and chronic respiratory failure. The process of interstitial inflamation goes fluently from alveolitis, through granuloma formation, to irreversible fibrosis.

In the last few years pneumoproteins (e.g. surfactant proteins) are considered to be markers of interstitial inflammation. In patients with idiopathic pulmonary fibrosis (IPF), it has been demonstrated that histological pattern of usual interstitial pneumonia (UIP) is related to low concentration of surfactant protein A (SP-A) and D (SP-D) in bronchoalveolar lavage (BAL) [1] and to high serum concentration of SP-A

Correspondence: W. Kucejko, Dept. of Lung Disease and Tuberculosis, Medical University of Białystok, Żurawia Str. 14, 15-540 Białystok, Poland; e-mail: wk_77@mp.pl. and SP-D. Serum concentration of SP-D is higher in patients with sarcoidosis than in healthy volunteers $[1,2]$. Similarly, the more active interstitial process in patients with hypersensivity pneumonitis (HP), the higher SP-D serum concentrations [3].

BAL concentration of Clara cell protein (CC-16) is decreased, and phospholipase A2 activity is increased in patients with IPF in comparison with healthy subjects [4]. It has been shown that in sarcoidosis serum CC-16 concentration is increased $[5,6]$, but CC-16 BAL concentration is similar to levels observed in healthy subjects.

This study was designed to determine the usefulness of determination biochemical markers (SP-D, CC-16, IL-10) in BAL derived from patients with sarcoidosis, IPF and HP. Another aim of this study was to answer the question whether SP-D, CC-16 and IL10 might be the markers of early stage of ILD activity and pulmonary fibrosis. 


\section{Materials and methods}

Patients. Bronchoalveolar lavage fluid was harvest from from 43 patients hospitalized and diagnosed in Pulmonary Department (Medical University of Białystok, Białystok, Poland) for suspicion of ILD. There were 27 patients with sarcoidosis (13 in II stage and 14 in III stage of sarcoidosis) 7 patients with IPF and 9 with HP. Patients did not present any signs of infection, or chronic lung disease. They were not treated with corticosteroids, immunosuppresive agents or any other drugs known as a potential ILD couse.

Patients were divided into 4 groups: group I - II stage of sarcoidosis, group II - III stage of sarcoidosis, group III - IPF, group IV - hypersensivity pneumonitis.

In every case laboratory tests (plasma $\mathrm{ACE}$ activity, plasma $\mathrm{Ca}$ concentration, $\mathrm{Ca}$ concentration in 24-hours urine collection), chest X-ray, chest high resolution computed tomography (HRCT), bronchofiberoscopy and BAL, spirometry, capillary blood gasometry, EKG, USG of abdominal organs, RT-23 were performed.

The diagnosis of sarcoidosis was made on the basis of histological presence of noncaseating granulomas, physical signs, bronchoscopic pattern, BAL, x-ray and HRCT. Radiological staging of sarcoidosis was carried out according to American Thoracic Society and European Respiratory Society criteria [7]. The disease activity was assessed on the grounds of BAL abnormalities, ACE plasma activity, Ca plasma and 24-hours urine colletion concentration. We also assessed organ involvement (heart, liver, spleen, kidneys and ocular involvement) in the course of sarcoidosis. Histological samples were collected during mediastiniscopy (4 cases), transbronchial lung biopsy (11 cases), bronchial mucus biopsy (6 cases), transbronchial lymph nodes biopsy (5 cases) and surgical lung biopsy (1 case).

Hypersensitivity pneumonitis was diagnosed according to HP diagnostic criteria [8]. In 6 cases transbronchial lung biopsy was performed to collect histological samples. Histological examinations revealed inflammatory cells infiltrations, and in one case, interalveolar septa fibrosis. In every case serological tests were performed to assess the presence of specific precipitins in serum.

Bronchofiberoscopy. Bronchoscopy was performed using Olympus BF-1T30 bronchofiberoscope. The patients were premedicated with midazolam $5 \mathrm{mg}$ and atropine $0.5 \mathrm{mg}$, followed by local anesthesia of upper and lower airways with $2 \%$ lidocaine solution.

Bronchoalveolar lavage (BAL). BAL was carried out according to criteria of ERS $[9,10]$. The bronchofiberoscope was inserted into the middle lobe or into the left lung lingual, alternatively. Lavage with $200 \mathrm{ml}$ of $0.9 \% \mathrm{NaCl}$ sterile solution $\left(37^{\circ} \mathrm{C}\right)$ was carried out by sequential instillation of four $50 \mathrm{ml}$ aliquots of saline. The BAL fractions were retrieved by gentle suction. The fluid recovery was calculated as the percentage of the instilled volume. The material was immediately sent to the laboratory. The BAL material was centrifuged (1000 rpm, $10 \mathrm{~min}$.). The total cell count and cell viability were calculated, as described before. The BAL filtrate samples were stored at $-20^{\circ} \mathrm{C}$ until assay performance.

The level of each marker was measured by commercially available specific kits according to the protocols provided by the manufacturer. SP-D, CC-16 and IL-10 BAL concentration were determined by ELISA method. SP-D and CC-16 kits came from BioVendor, Brno, Czech Republic. The test sensitivity was $0.5 \mathrm{ng} / \mathrm{ml}$. IL-10 kit came from R\&D Systems, Minneapolis, USA. The test sensitivity was $<3.9 \mathrm{pg} / \mathrm{ml}$. The assays were performed simultaneously, after samples defrosting. For each patient's sample two assays were performed, and the mean value was considered as the final value.

Statistical analysis. All measured values were presented as medians \pm SEM (due to nonparametric distribution of values). Qualita- tive traits were presented as quantitative-percentage distribution. The Mann-Whitney U-test was used to compare the data obtained in analysed groups. The Spearman's rank correlation coefficient rs was applied to test the correlation between two random variables. $\mathrm{P}$-values $<0.05$ were considered as statistically significant. P-values $>0.05$ and $<0.1$ were considered to be within statistical significance limit.

\section{Results}

Basic patients and groups characteristics are shown in Table 1. In group III (patients with IPF) the VC values were significantly lower than in group I (patients with II stage of sarcoidosis) and than in group II (patients with III stage of sarcoidosis) $(\mathrm{p}=0.014$ and $\mathrm{p}=0.002$, respectively). The highest mean $\mathrm{FEV}_{1}$ value (97.92\% expected) was found in group I, and the lowest $(76.99 \%$ expected) - in group III. The highest mean VC value $(96.83 \%)$ was found in group II, and the lowest $(70.23 \%)$ in group III. The serum ACE activity (73.15 U/1) was the highest in patients with II stage of sarcoidosis.

We found the highest mean BAL cytosis in patients with II stage of sarcoidosis and in patients with hypersensivity pneumonitis - in both groups 1.5 $\times 10^{6} / \mathrm{ml}$. The lowest BAL cytosis $\left(1.43 \times 10^{6} / \mathrm{ml}\right)$ was present in patients with IPF. In group I and II we found statistically significant higher BAL limphocytosis than in group III ( $\mathrm{p}=0.015$ and $\mathrm{p}=0.026$, respectively). There was a higher neutrophil BAL percentage in patients with IPF than in patients with II stage of sarcoidosis $(\mathrm{p}=0.008)$ and in patients with hypersensivity pneumonitis $(\mathrm{p}=0.032)$. Similarly, in IPF group the BAL percentage of eosinophils was higher than in group I, II and IV ( $\mathrm{p}=0.021, \mathrm{p}=0.014$ and $\mathrm{p}=0.002$, respectively).

The mean values of capillary blood gasometry were close to normal, there were no significant differences between groups.

We observed the highest SP-D BAL concentration in patients with the III stage of sarcoidosis $(96.67 \mathrm{ng} / \mathrm{ml})$, and the lowest in patients with IPF $(76.49 \mathrm{ng} / \mathrm{ml})$. Similarly, the BAL concentration of Clara cell protein was the highest in patients with III stage of sarcoidosis $(31.78 \mathrm{ng} / \mathrm{ml})$, and the lowest in patients with hypersensivity pneumonitis $(21.39 \mathrm{ng} / \mathrm{ml})$ (Fig. 1). The concentration differences were not statistically significant.

The highest BAL concentration of IL-10 was found in patients with IPF $(2.04 \mathrm{pg} / \mathrm{ml})$; the lowest in patients with hypersensivity pneumonitis (1.68 $\mathrm{pg} / \mathrm{ml}$ ). In IPF group IL-10 concentration was significantly higher than in the group of patients in III stage of sarcoidosis $(p=0.02)$ and in the HP group $(p=0.023)$. In comparison to patients with II stage of sarcoidosis, the difference was within statistical significance limit $(\mathrm{p}=0.08)$. 
Table 1. Baseline patients characteristics

\begin{tabular}{|c|c|c|c|c|}
\hline Characteristics & III stage of sarcoidosis & III stage of sarcoidosis & $\mathrm{IIP}$ & IPl: \\
\hline Patients & 13 & 14 & 9 & 7 \\
\hline Age (years) & $35.5 \pm 12.0$ & $40.6 \pm 8.3$ & $42.0 \pm 12.7$ & $51.6 \pm 11.7$ \\
\hline Women / Men & $6 / 7$ & $7 / 7$ & $0 / 9$ & $4 / 3$ \\
\hline Non-smokers / Smokers & $12 / 1$ & $14 / 0$ & $9 / 0$ & $7 / 0$ \\
\hline FEV1 (\% predicted value) & $94.4 \pm 24.2$ & $96.7 \pm 16.9$ & $88.9 \pm 28.6$ & $77.0 \pm 22.7$ \\
\hline VC (\% predicted value) & $91.4 \pm 17.0$ & $97.8 \pm 13.2$ & $87.6 \pm 24.2$ & $70.73 \pm 17.1$ \\
\hline Total cells - BAL cytosis $\left(\times 10^{6}\right)$ & $1.5 \pm 0.5$ & $1.5 \pm 0.6$ & $1.5 \pm 0.6$ & $1.4 \pm 0.4$ \\
\hline BAL macrophages (\%) & $48.3 \pm 16.0$ & $48.6 \pm 19.5$ & $56.3 \pm 17.7$ & $54.1 \pm 18.7$ \\
\hline BAL lymphocytes (\%) & $48.6 \pm 16.3$ & $46.6 \pm 18.4$ & $39.1 \pm 20.8$ & $26.7 \pm 22.7$ \\
\hline BAL neutrophils (\%) & $2.2 \pm 4.8$ & $3.7 \pm 6.1$ & $1.3 \pm 0.9$ & $9.3 \pm 7.4$ \\
\hline BAL eosinophils (\%) & $0.9 \pm 0.8$ & $0.9 \pm 0.8$ & $3.2 \pm 6.3$ & $9.86 \pm 6.6$ \\
\hline $\mathrm{pCO}_{2}(\mathrm{mmHg})$ & $37.7 \pm 3.2$ & $34.1 \pm 4.6$ & $33.7 \pm 6.3$ & $36.0 \pm 4.3$ \\
\hline $\mathrm{pO}_{2}(\mathrm{mmHg})$ & $72.0 \pm 9.5$ & $66.7 \pm 10.0$ & $68.2 \pm 5.4$ & $66.2 \pm 7.2$ \\
\hline $\mathrm{O}_{2}$ saturation (capillary blood gasometry) & $94.7 \pm 1.9$ & $93.1 \pm 2.3$ & $93.7 \pm 1.2$ & $93.7 \pm 1.7$ \\
\hline ACE serum activity $(\mathrm{U} / \mathrm{l})$ & $69.0 \pm 41.7$ & $71.9 \pm 33.5$ & $46.2 \pm 26.4$ & - \\
\hline $\mathrm{Ca}$ in 24-hours urine collection & $4.3 \pm 1.6$ & $3.3 \pm 1.4$ & $3.6 \pm 1.1$ & - \\
\hline Serum Ca concentration & $2.3 \pm 0.2$ & $2.3 \pm 0.2$ & $2.2 \pm 0.1$ & - \\
\hline
\end{tabular}

HP - hypersensivity pneumonitis; IPF - idiopathic pulmonary fibrosis; FEV1 - forced expiratory volume in one second; VC - vital capacity; BAL

- broncho-alveolar lavage; ACE - agiotensin converting enzyme

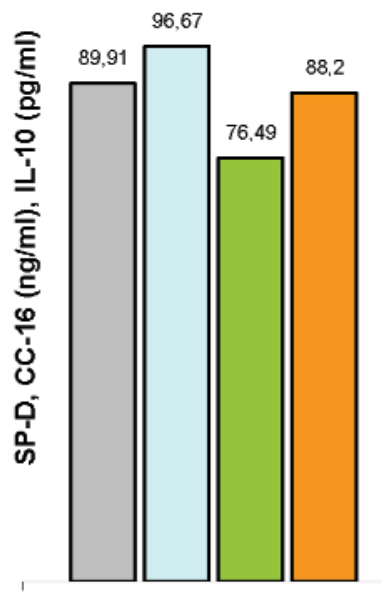

SP-D

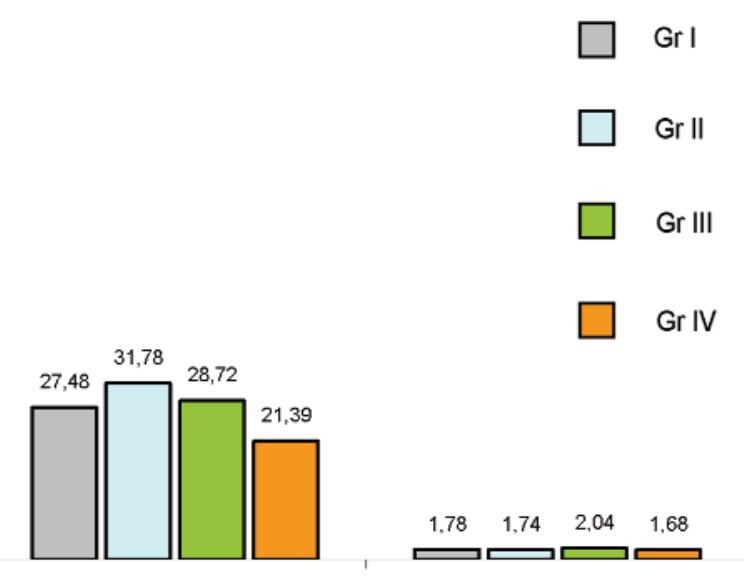

IL-10
Fig. 1. BAL concentration of SP-D, CC-16 and IL-10 in different groups. SP-D - surfactant protein D; CC-16 Clara cell protein; BAL - bronchoalveolar lavage

\section{Correlations in group I (II stage of sarcoidosis)}

Higher BAL SP-D concentrations were related to lower $\mathrm{VC}$ values $(\mathrm{p}=0.07), \mathrm{FEV}_{1}$ values and lower lymphocytes percentage in BAL $(p=0.09)$. Higher BAL SP-D levels were related to higher percentage of BAL neutrophils $(p=0.076)$, and to lower BAL cytosis. We found statistically significant correlation between BAL CC-16 concentration and plasma ACE activity (Fig. 2).

\section{Correlations in group II (III stage of sarcoidosis)}

We found that the higher IL-10 concentrations, the higher SP-D concentrations ( $\mathrm{p}=0.056$; Fig. 3 ). The same relationship was observed between IL-10 and CC-16 concentration ( $\mathrm{p}=0.048$; Fig. 4 ). The important thing is that low SP-D BAL concentrations were related to low $\mathrm{FEV}_{1}$ and $\mathrm{VC}$ values. The second dependence was statistically significant ( $\mathrm{p}=0.038$; Fig. 5 ). In this group high SP-D concentration was associated 


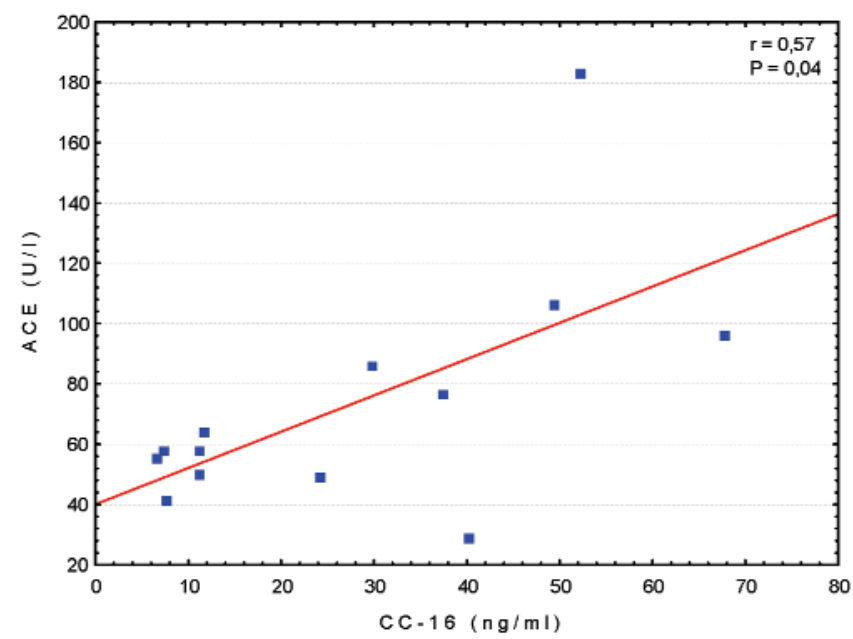

Fig. 2. Relationship between BAL concentration of CC-16 and serum activity of ACE in group I. ACE - angiotensin converting enzyme; CC-16 - Clara cell protein; BAL - bronchoalveolar lavage

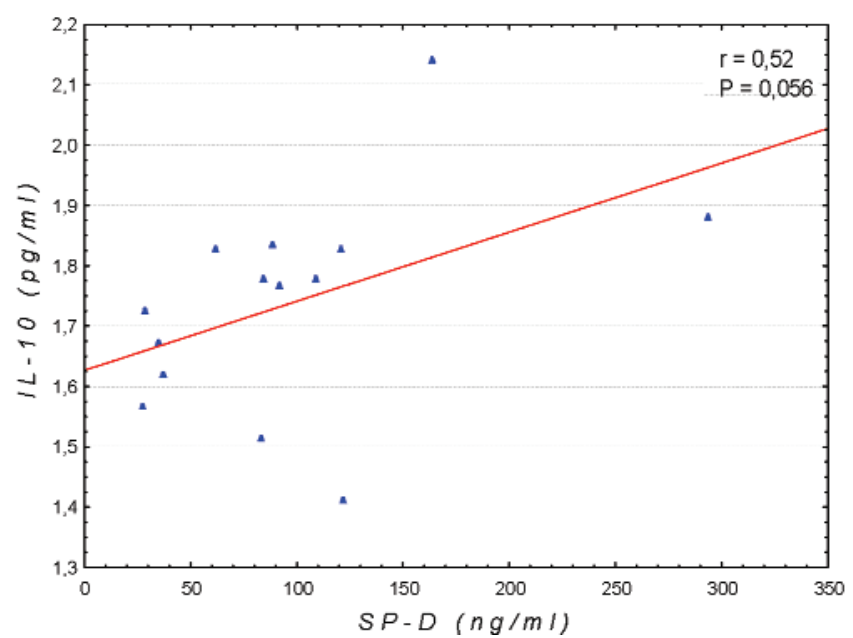

Fig. 3. Relationship between BAL concentrations of SP-D and IL10 in group II. SP-D - surfactant protein D; BAL - bronchoalveolar lavage; IL-10 - interleukin 10

with high BAL cytosis and high BAL percentage of lymphocytes, neutrophils and eosinophils. We also found that high SP-D level was related to low $\mathrm{pO}_{2}$ values.

\section{Correlations in group III (IPF)}

High CC-16 concentration was related to lower BAL cytosis and to lower BAL neutrophils percentage. In this case there was a higher BAL percentage of lymphocytes and eosinophils. High SP-D concentration was related to lower BAL percentage of lymphocytes, neutrophils and eosinophils. We also found, that higher CC-16 concentrations were related to higher $\mathrm{pO}_{2}$ values and to a higher blood $\mathrm{O}_{2}$ saturation. In this

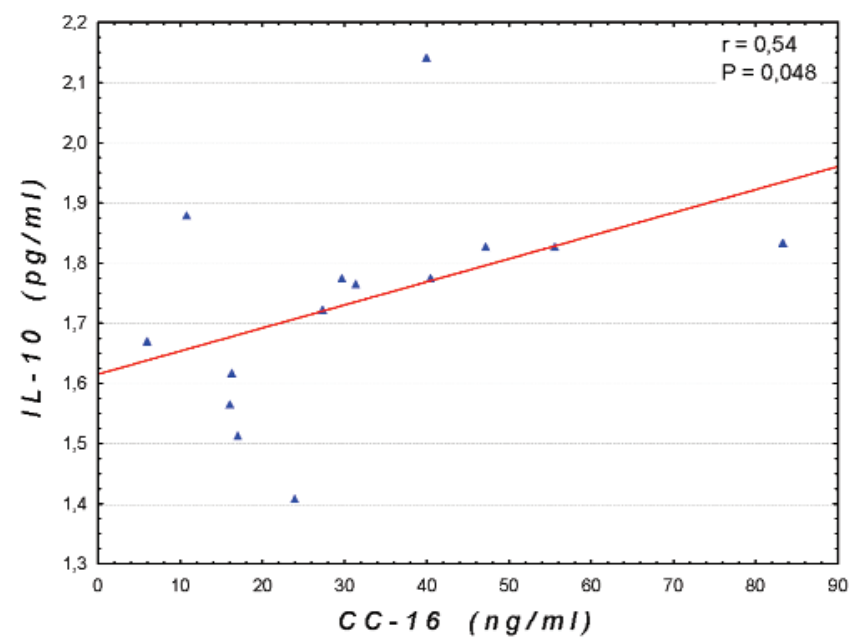

Fig. 4. Relationship between BAL concentrations of IL-10 and CC-16 in group II. IL-10 - interleukin 10; CC-16 -Clara cell protein; BAL - broncholaveolar lavage

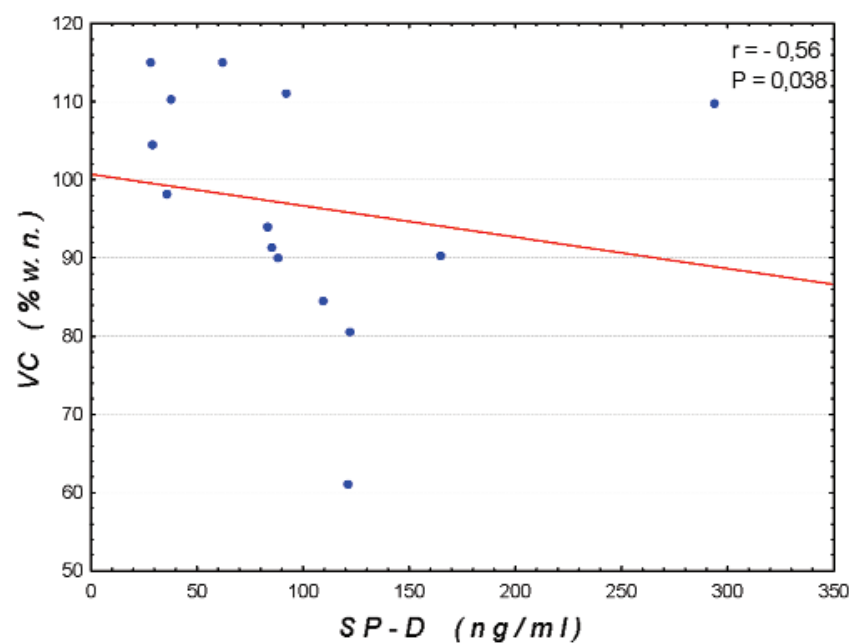

Fig. 5. Relationship between VC and BAL concentration of SP-D (group II). VC - vital capacity; SP-D - surfactant protein D; BAL - bronchoalveolar lavage; w.n. - percentage of expected value

group we did not find any correlations between BAL concentration of SP-D nor IL-10 and the lung function tests abnormalities. But high CC-16 concentrations were related to low $\mathrm{VC}$ values. We observed, that the higher SP-D levels, the higher $\mathrm{pCO}_{2}$ and the lower $\mathrm{pO}_{2}$ in capillary blood gasometry. According to our expectations, higher IL-10 levels were related to lower BAL cytosis and to lower percentages of neutrophils and eosinophils.

\section{Correlations in group IV (Hypersensivity pneumonitis)}

Higher SP-D concentration was related to higher IL-10 and lower CC-16 levels. Similarly, lower SP-D levels 
were associated with lower $\mathrm{FEV}_{1}$ and $\mathrm{VC}$ levels, as in group II. Moreover, the higher SP-D concentration, the lower BAL cytosis and the lower BAL percentage of lymphocytes, neutrophils and eosinophils. We also observed that, the higher SP-D level, the higher serum ACE activity (not statistically significant).

\section{Discussion}

Bronchoalveolar lavage (BAL) allows researchers to investigate the processes going on in alveoli. Both cellular and liquid BAL components can be studied (e.g. pneumoproteins) $[6,11,12]$.

There are not many publications on SP-D BAL concentrations in healthy volunteers. Mean SP-D concentration according to different authors ranges from about 0.9 to $1.3 \mathrm{mg} / 1[1,13]$. Mean CC-16 concentration in BAL from healthy volunteers is about 0.1 to $3.6 \mathrm{mg} / 1[5,14,15,16]$. The differences might result from usage of different monoclonal antibodies and different standards by research centers. It concerns both patients and healthy volunteers [7].

According to Ishii et al. [18] inflammation process engaging alveoli results in destruction of II type pneumocytes and other cells lining both alveoli and bronchioles. Above-mentioned cell contents (e.g. surfactant proteins) is released into alveoli lumen. Protracting inflammation process leads to granuloma formation and to partial regenerating of alveolar lining cells. Alveolar type II cells become hyperplastic. At the same time fibroblast migration starts, and subsequently extracellular matrix deposition begins together with interstitial fibrosis. This process might lead to lung parenchyma remodeling and to developing "honeycomb change" on HRCT scans. Then, surfactant proteins concentration decreases. It happens because of both reduced synthesis and increased phagocytosis by alveolar macrophages $[18,19]$.

In the course of sarcoidosis and IPF higher serum levels of CC-16 were observed. Little is known on CC16 concentration in BAL fluid. To date is has been shown that in BAL from smokers the level of this protein is decreased. The reason for this observation might be the decreased number of Clara cells $[16,20,21]$. More over, in healthy subjects there is a higher BAL concentration of CC-16 than in patients with IPF, in patients with asbestosis and than in subjects with ARDS $[22,23,24]$. In those cases the most reasonable explanation of decreased CC-16 level was unfavourable course of the disease.

In this study, we compared the value of SP-D, CC16 and IL-10 as BAL markers of disease activity in different ILDs - sarcoidosis, idiopathic pulmonary fibrosis and hypersensivity pneumonitis. Mean BAL levels that we found were lower than published by other authors. We observed the highest SP-D concen- tration in patients with III stage of sarcoidosis. Lower SP-D concentrations were related to higher VC values in patients with II stage of sarcoidosis, in contrast with patients with III stage of sarcoidosis. In both groups mean $\mathrm{VC}$ values were close to normal. In agreement to previous studies [25], higher levels of SP-D were related to higher BAL cytosis and higher percentages of BAL lymphocytes. In this study, the higher CC-16 level, the higher ACE serum activity. Other authors, e.g. Janssen [2], determined serum pneumoproteins concentrations, and compared their results with those from healthy subjects. Such studies demonstrated higher concentrations of SP-D and CC16 in both sarcoidosis and IPF as compared to healthy volunteers. The more lung parenchyma involvement in sarcoidosis, the higher concentrations of studied proteins $[2,25]$.

Previous studies $[1,3,18]$ demonstrated similar findings to ours for SP-D in IPF. In IPF in contrast with the III stage of sarcoidosis, higher levels of SP-D were related to lower BAL percentage of lymphocytes. In might result from different pathogenesis of the above-mentioned diseases.

In this study we demonstrated the reverse correlation between SP-D and CC-16. To our knowledge there are no studies comparing BALF concentration of these pneumoproteins in patients with IPF. In the course of idiopathic pulmonary fibrosis lung parenchyma remodeling develops. It might be the reason for decreased number of Clara cells, and in consequence the reason for low CC-16 BALF concentration.

Higher SP-D, CC-16 and IL-10 concentrations were related to lower BAL percentage of lymphocytes, neutrophils and eosinophils. These findings are in agreement with expectations, because of the antiinflammatory properties of studied pneumoproteins. But the recent data show no evidence of inflammation etiology in the pathogenesis of IPF [26,27]. The latest theory describes repeated episodes of destruction of lung epithelium and abnormal reconstructive processes leading to fibrinogen deposition in the extracellular space [28]. It seems that different intensification of typical histopathological changes in IPF (alveolitis, fibroblast foci separated by normal lung interstitium, foci of fibrosis) might be the reason for discrepancy in recent reports [17].

We demonstrated the lowest concentrations of CC16 and IL-10 in patients with hypersensivity pneumonitis. In this group SP-D levels were higher than in IPF group and lower than in both sarcoidosis groups. Higher SP-D levels, similarly to IPF, were related to lower CC-16 levels. In the course of hypersensivity pneumonitis decreased number of Clara cells accompanied by low levels of CC-16 might be also observed. In our HP group there were patients with acute, subacute and chronic form of the disease. Similarly to IPF 
patients, we found in HP that higher SP-D concentrations were related to lower BAL cytosis, lower BAL percentage of lymphocytes, neutrophils and eosinophils. Despite different pathogenesis, the reason for the above-mentioned relationships seems to be the same - anti-inflammatory properties of this protein. In acute hypersensivity pneumonitis with active granuloma formation process higher SP-D concentrations related to higher ACE serum activity, just as in sarcoidosis.

In conclusion, we found higher level of SP-D in BAL fluid in active interstitial inflammation with larger parenchyma engagement (III stage of sarcoidosis), than in chronic stage of interstitial inflammation (IPF). Moreover, we found the highest concentration of CC16 in patients with III stage of sarcoidosis, suggesting destruction of Clara cells. Because of the small number of patients in each group further studies on pneumoproteins focusing on their role in the pathogenesis of ILDs and in pulmonary fibrosis are necessary.

\section{References}

[1] Honda Y et al. Pulmonary surfactant protein D in sera and bronchoalveolar lavage fluids. Am J Respir Crit Care Med. 1995;152:1860-1866.

[ 2] Janssen R et al. Study of Clara cell 16, KL-6, and surfactant protein $\mathrm{D}$ in serum as disease markers in pulmonary sarcoidosis. Chest. 2003;124:2119-2125.

[3] Ohnishi H et al. Comparative study of KL-6, surfactant protein $\mathrm{A}$, surfactant protein $\mathrm{D}$, and monocyte chemoattractant protein 1 as serum markers for interstitial lung disease. $\mathrm{Am} \mathrm{J}$ Respir Crit Care Med. 2002;165:378-381.

[4] Miele L. et al. Uteroglobin: structure, molecular biology, and new perspectives on its function as a phospholipase A2 inhibitor. Endocr Rev. 1987;8:474-490.

[5] Bernard A et al.: Clara cell protein in serum and bronchoalveolar lavage. Eur Respir J. 1992;5:1231-1238.

[ 6] Hermans C. et al. Lung epithelium specific proteins: characteristics and potential applications as markers. Am J Respir Crit Care Med. 1999;159:646-678.

[7] American Thoracic Society/European Respiratory Society. Statement on sarcoidosis. Am J Respir Crit Care Med. 1999;160:736-755.

[8] Patel AM et al. Hypersensivity pneumonitis: Current concepts and future questions. J Allergy Clin Immunol. 2001;108(5):661-670.

[9] Klech $\mathrm{H}$ et al. Clinical guidelines and indications for bronchoalveolar lavage (BAL): Report of the European Society of Pneumonology Task Group on BAL. Eur Respir J. 1990;3:937-974.

[10] Klech $\mathrm{H}$ et al. Technical recommendations and guidelines for BAL. Report of the European Society of Pneumonology Task
Group on BAL. Eur Respir J. 1989;2:561-585.

[11] Ryu JH, Daniels CE, Hartman TE et al. Diagnosis of interstitial lung diseases. Mayo Clin Proc. 2007;82(8):976-86.

[12] Tötsch M, Guzman J, Theegarten D, et al.: Bronchoalveolar lavage. Pathologie. 2007;29.

[13] Honda Y et al. Decreased contents of surfactant proteins A and $\mathrm{D}$ in BAL fluids of healthy smokers. Chest. 1996;109:1006-1009.

[14] Bernard A et al. The molecular mass and concentrations of protein 1 or Clara cell protein in biological fluids. Clin Chim Acta. 1993;223:189-191.

[15] Ishii S et al. Protein 1: its purification and application in clinical medicine. J Clin. Lab. Anal. 1993;7:394-400.

[16] Shijudo N et al. Serum and BAL Clara cell protein levels, and CC-10-positive bronchiolar cells are decreased in smokers. Eur Respir J. 1997;10:1108-1114.

[17] Hermans C. et al. Serum Clara cell protein (CC-16) correlates with chest radiographic abnormalities of sarcoidosis. Am J Respir Crit Care Med. 1997;155:981.

[18] Ishii $\mathrm{H}$ et al. High serum concentration of surfactant protein A in usual interstitial pneumonia compared with non-specific interstitial pneumonia. Thorax. 2003;58:52-57.

[19] Greene KE et al. Serum surfactant protein A and D as biomarkers in idiopathic pulmonary fibrosis. Eur Respir J. 2002; 19:439-446.

[20] Lumsden AB et al. Goblet and Clara cells of human distal airways: evidence for smoking induced changes in their numbers. Thorax. 1984;39:844-849.

[21] Nomori $\mathrm{H}$ et al. Clinicopathological examination of the relation between Clara cells and smoking. Kyobu Geka. 1994; 47:888-891.

[22] Jorens PG et al. Potential role of of Clara cell protein, an endogenous phpspholipase-A2 inhibitor, in acute lung injury. Eur Respir J. 1995;8:1647-1653.

[23] Lesur O et al. Clara cell protein (CC-16) and surfactant-associated protein A (SP-A) in asbestos-exposed workers. Chest. 1996;109:467-474.

[24] Lesur O et al. Clara cell protein (CC-16) induces a phospholipase A2-mediated inhibition of fibroblast migration in vitro. Am J Respir Crit Care Med. 1995;152:290-297.

[25] Kunitake R et al. KL-6, surfactant protein A and D in bronchoalveolar lavage fluid from patients with pulmonary sarcoidosis. Respiration. 2001;68:488-495.

[26] American Thoracic Society/European Respiratory Society International Multidisciplinary Consensus Classification of the Idiopathic Interstitial Pneumonias. Am. J. Respir. Crit Care Med. 2002;165:277-304.

[27] American Thoracic Society/European Respiratory Society. Idiopathic pulmonary fibrosis: diagnosis and treatment. International consensus statement. Am. J. Respir. Crit. Care Med. 2000;161:646-664

[28] Wesołowski S: Idiopatyczne śródmiąższowe zapalenia płuc. In: Rowińska-Zakrzewska E, ed. Choroby uktadu oddechowego. PZWL, Warszawa; 2004:221-237.129.

Submitted: 24 June, 2008 Accepted after reviews:16 November, 2008 